\title{
APPLICATION OF DUAL IONIC/COVALENT CROSSLINKING IN LECITHIN/ CHITOSAN NANOPARTICLES AND THEIR EVALUATION AS DRUG DELIVERY SYSTEM
}

\author{
ISRA DMOUR* and HASAN MUTI
}

${ }^{1}$ Department of Pharmaceutics and Pharmaceutical Technology,

\author{
Pharmacological and Diagnostic Research Center (PDRC), Faculty of Pharmacy, \\ Al-Ahliyya Amman University, Amman, Jordan
}

\begin{abstract}
The aim of this study was to investigate the use of chitosan derivatives in the formulation of lecithin/chitosan (LEC/CHI) nanoparticles (NPs), and to evaluate them as drug delivery systems after the application of dual ionic/covalent crosslinking. Chitosan and two chitosan conjugates were investigated in this study: Chitosan Phthalate and Phenylsuccinate with two degrees of substitution were used: 20 and $40 \%$. All NPs were characterized by hydrodynamic diameter, zeta potential, $\mathrm{pH}$ stability, drug loading, and encapsulation efficiency, and in vitro release studies. Metronidazole (MET) was used as a model drug in the evaluation of drug-loaded NPs. The drug-free NPs before and after crosslinking have a size of less than $100 \mathrm{~nm}$ irrespective of the type of chitosan used. The zeta potential ranged between 14 and $23 \mathrm{mV}$ depending on the type of chitosan and the crosslinker used. The MET-loaded NPs showed an increase in hydrodynamic diameter reaching up to $400 \mathrm{~nm}$ in some formulas. It was found that NPs based on chitosan derivatives showed higher drug loading capacities when compared to unmodified chitosan. Following the application of dual crosslinking, the drug loading of MET ranged between 9 and $26 \%$ while the entrapment efficiency ranged between 56 and $73 \%$. Release studies showed higher release amounts for dual crosslinked NPs with the exception of the phenylsuccinic chitosan $20 \%$ formula. The use of chitosan derivatives and the dual crosslinking process offers more promising capabilities compared to the classical $\mathrm{LEC} / \mathrm{CHI} \mathrm{NP}$ assembly in relation to drug loading and release properties.
\end{abstract}

Keywords: lecithin, chitosan, nanoparticles, covalent Crosslinking, metronidazole, release

Nano drug delivery systems have interested many researchers in the last decade due to their promising properties compared to conventional drug carriers. Nanoparticles (NPs) have many applications in the medical and pharmaceutical fields. NPs and other colloidal drug-delivery systems modify the kinetics, body distribution, and release of an associated drug. NPs have been developed as an important approach for the delivery of conventional drugs, recombinant proteins, vaccines, and more recently, nucleotides (1). Among nanoparticulate systems that show a promising future are lecithin /chitosan (LEC/CHI) based NPs, which were first introduced by Sonvico et al. as drug delivery systems to deliver lipophilic drugs (2). Lecithin (LEC), a soya-based phospholipid consisting of phosphatidylcholine and phosphatidylethanolamine, is considered a safe substance due to its biodegradability and is frequently used for the preparation of liposomal drug delivery systems (Figure 1). On the other hand, chitosan $(\mathrm{CHI})$ is a polysaccharide polymer derived from chitin and consisting of glucosamine with potentially biocompatible non-toxic, and biodegradable properties. This biopolymer has been used as a pharmaceutical excipient due to its binding and bioadhesive properties. Chitosan-based NP systems have been widely investigated for the delivery of antibiotics, anti-cancer, and many other drugs via several routes of administrations including parenteral, intranasal, dermal, etc. (3-5).

LEC/CHI-based NPs have been investigated to deliver drugs via nasal, ophthalmic, and parenteral routes for many drugs like insulin, melatonin, and clobetasol, etc. (6-9). LEC/CHI NPs are prepared by electrostatic interaction between the negatively charged lecithin and the positively charged amines of CHI. They offer many advantages including

* Corresponding author: e-mail: isradmdm@gmail.com 
improved bioavailability and efficient delivery of poorly water-soluble compounds compared to other polymers, due to the presence of phospholipid bilayers within the NP assembly (10). However, one drawback of the LEC/CHI NP system is that only hydrophobic molecules such as progesterone can be loaded in this assembly, since loading capacities are usually decreased for hydrophilic drugs (11).

Among the main properties to be considered during NP formulation are the release and loading properties, as they greatly affect the amount of drug reaching the active site. Various techniques have been employed to enhance the release including physico-chemical manipulation of the drug or the nanocarrier. One of the attractive properties of chitosan is that it can be chemically modified by introducing functional groups that can affect its gelation, mechanical properties, loading capacity, and permeability. Several reports have been published describing chitosan conjugation including methylation, carboxylation, thiolation, etc. $(5,11)$. The idea of dual crosslinking of polymeric NPs has been previously reported to enhance their drug release and stability. Polymeric crosslinking involves the connection between the polymeric chains via ionic or covalent bonding. Various crosslinkers have been used including tripolyphosphates, carbodiimide, glutaraldehyde, etc. A few reports have been published in this regard, for example using ionic gelation followed by covalent crosslinking to deliver doxorubicin (11-12). The application of covalent crosslinking by using controlled oxidation with hydrogen peroxide $\left(\mathrm{H}_{2} \mathrm{O}_{2}\right)$ in chitosan thioglycolic acid-based NPs for mucosal drug delivery was also described by Barthelmes et al. (13).

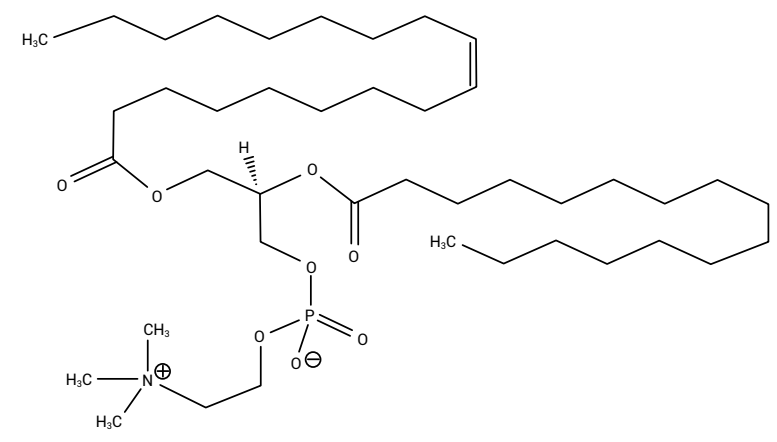

Lecithin<smiles>Cc1ncc([N+](=O)[O-])n1CCO</smiles>

Metronidazole<smiles>CP(=O)(O)OP(=O)(O)OP(=O)(O)O</smiles>

Tripolyphostate (TPP)

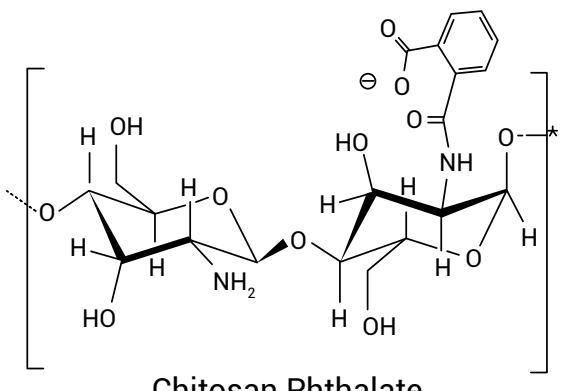

Chitosan Phthalate

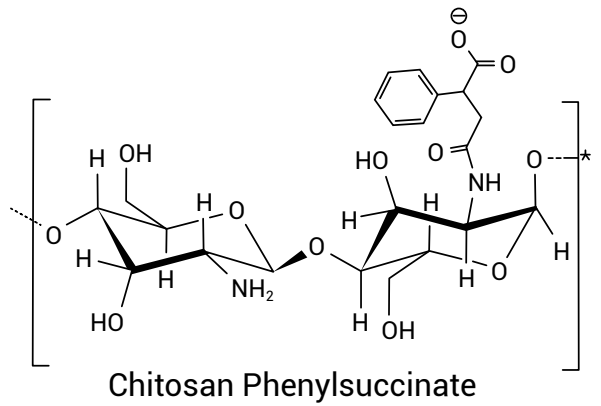

Chitosan Phenylsuccinate 
A similar approach was adopted by Soliman et al. in the stabilization of a chitosan-hydrocaffeic acid conjugate (14). Recently, Vila-Sanjurjo et al. used dual crosslinking by genipin and sodium tripolyphosphate (TPP) to investigate the ability of chitosan NPs to target bacterial populations (15).

In this article, we investigate the use of chitosan conjugate instead of chitosan in the preparation of lecithin-based NPs using two dicarboxylic acids (phthalate (PH) and phenylsuccinate conjugate (PS)) with two Degrees of Substitution (DS), 20 and $40 \%$. In addition, we applied dual ionic crosslinking, using tripolyphosphate (TPP), and covalent crosslinking, using Carbodiimide coupling, to these NPs, and compared it to the conventional LEC/CHI NPs. Here, we evaluated the crosslinking in each step by measuring the hydrodynamic diameter and zeta potential. We anticipated that dual crosslinking would improve the loading and release properties. Metronidazole (MET), an anti-protozoal, was used as a model drug with moderate solubility in the loaded NPs. MET (Figure 1) contains both lipophilic and hydrophilic functional groups with slight water-solubility properties (16).

\section{EXPERIMENTAL}

\section{Materials}

In this study, we used medium molecular weight chitosan, phenylsuccinic anhydride and N-ethyl$\mathrm{N}^{\prime}$-(3-dimethylaminopropyl) carbodiimide hydrochlorides (EDC) (Sigma-Aldrich, USA). phthalic anhydride (Fluka, Switzerland). Penta basic sodium tripolyphosphate (TPP) and TRIS base buffer (Sigma-Aldrich, Germany). We used dialysis tubing (molecular weight cutoff $=12 \mathrm{kDa}$, SigmaAldrich, USA). Metronidazole (MET) was used as a model drug, thanks to a generous gift from Dar Al-Dawa (DAD) Pharmaceutical Manufacturing company (Naor, Jordan). Ultrapure water (conductivity $=0.05 \mu \mathrm{s} / \mathrm{cm}$ ) was used for DLS size analysis (Millipore, USA). All other materials and solvents were of analytical grades.

\section{Synthesis of chitosan conjugates}

Chitosan conjugates (Chitosan Phthalate and Chitosan Phenylsuccinate) were prepared as described earlier $(11,17)$. with slight modifications. Briefly, chitosan (1.00 g, corresponding to $5.58 \mathrm{mmol}$ glucosamine) was dissolved in $(50 \mathrm{~mL})$ aqueous solution $\mathrm{HCl}(0.37 \% \mathrm{v} / \mathrm{v})$. The particular anhydride (phthalic or phenylsuccinic acids) was dissolved in $(5 \mathrm{~mL})$ pyridine added dropwise to chitosan solution with vigorous stirring. Two degrees of substitution were prepared (20 and 40\%) for each chitosan derivative. The $\mathrm{pH}$ of the solution was maintained at 7.0 using $1 \mathrm{M} \mathrm{NaOH}$ solution. The reaction was allowed to continue for $40 \mathrm{~min}$ with continuous stirring. The reaction was terminated by the gradual addition of acetone under continuous stirring resulting in precipitation of the chitosan derivative. Following filtration, the precipitate was washed three times with $100 \mathrm{~mL}$ absolute ethanol, and finally with acetone $(100 \mathrm{~mL})$, and dried for $48 \mathrm{~h}$ in a hot air oven at $45^{\circ} \mathrm{C}$. The products were stored in airtight bottles until further use.

\section{Fabrication of lecithin/chitosan or chitosan derivatives nanoparticles}

Lecithin/chitosan and chitosan derivative NPs were prepared according to the method described by Sonvico et al. with slight modifications (2). Chitosan was dissolved in $0.275 \mathrm{~N} \mathrm{HCl}$ to get $0.5 \%(\mathrm{w} / \mathrm{v})$ concentration. Freshly prepared lecithin $(2.5 \% \mathrm{w} / \mathrm{v}$ in $96 \%$ ethanolic solution) was always used. Two proportions of lecithin to chitosan were used in this study (10:1 and $5: 1)$. NPs were generated spontaneously by dropwise addition of $4 \mathrm{~mL}$ of ethanolic solution of lecithin in $46 \mathrm{~mL}$ aqueous solution of chitosan under magnetic stirring using the syringe method (the syringe having an internal diameter of $0.38 \mathrm{~mm}, 1 \mathrm{~mL} / \mathrm{min}$ injection rate). Following the appearance of a hazy solution, the stirring was allowed to continue for $2 \mathrm{~min}$. NPs based on chitosan conjugates were prepared with the same method and proportions. In the preparation of drug-loaded NPs, the ratio of $1: 1$ lecithin to the drug was selected for evaluation (equivalent to a dose of $2 \mathrm{mg}$ MET per $1 \mathrm{~mL}$ NP dispersion). MET was dissolved in the chitosan solution before starting the dropping method. Drug-free NPs were prepared as previously described and were used for comparison.

\section{Dual ionic/covalent crosslinking of the prepared nanoparticles using TPP/EDC}

Aliquots of $5 \mathrm{~mL}$ of the preparation of LEC/ CHI NPs were placed in vials, and the respective amount of TPP $(0.4 \mathrm{w} / \mathrm{v} \%)$ was added (data from a previous study (11) with continuous stirring for $2 \mathrm{~min}$. This was followed by the addition of $10 \mathrm{mg}$ EDC to each vial and again stirring was continued for $2 \mathrm{~min}$ (Figure 2). For size and zeta potential evaluations, the aliquots were dialyzed against water before measurement.

\section{Characterization of nanoparticles Particle size and zeta potential measurements}

The hydrodynamic diameter (z-average) and polydispersity index (PDI) of the NPs were 

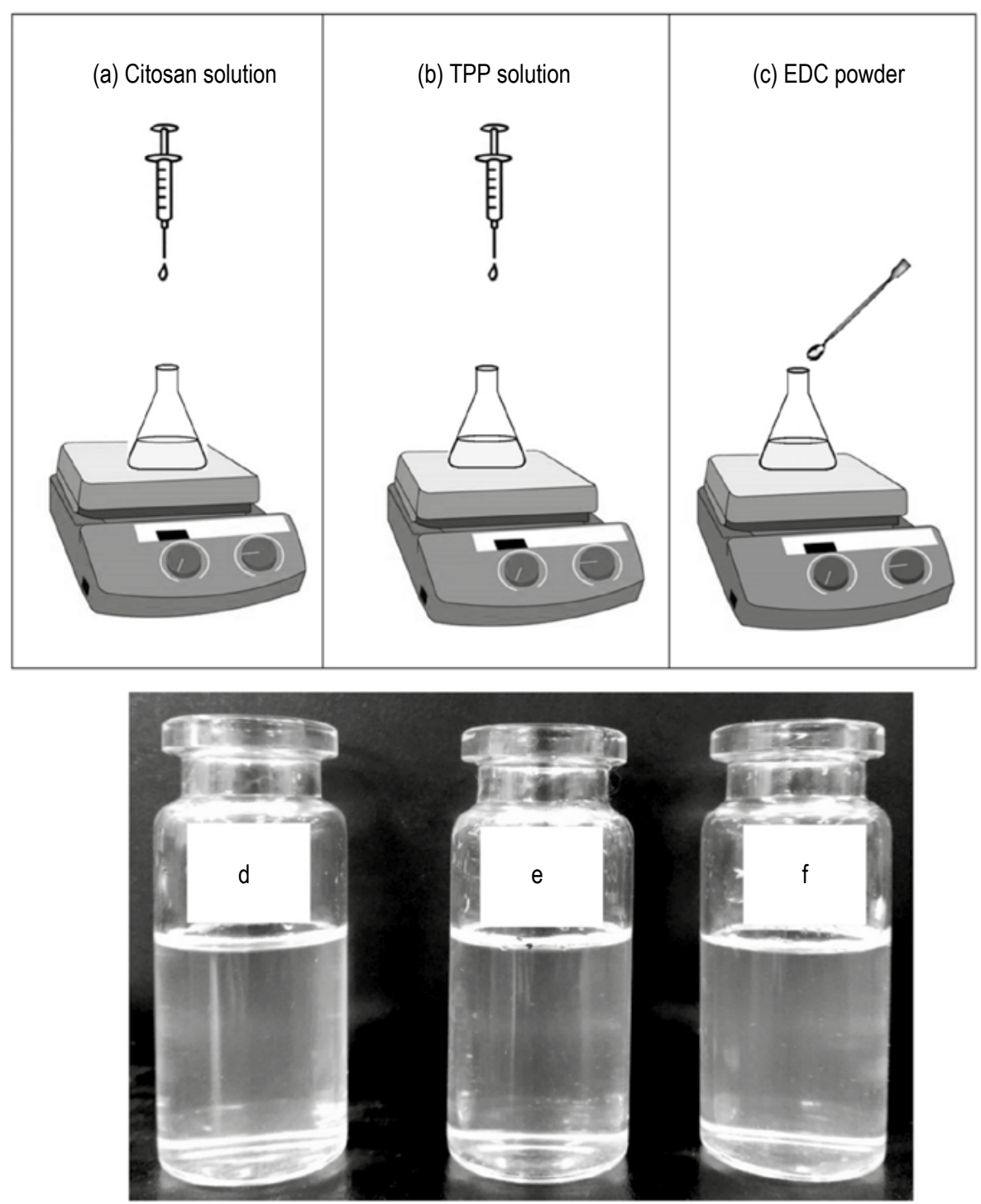

Figure 2. Above: Scheme of the experimental set-up for crosslinking process: (a) Addition of Chitosan to Lecithin solution (b) addition of TPP dropwise (c) addition of EDC powder with continuous stirring. Below: Visual observation of (d) Lecithin/Chitosan Phthalate 40\% NPs (e) Lecithin/Chitosan Phthalate-TPP NPs 40\% and (f) Lecithin/Chitosan phthalate TPP-EDC 40\%.

determined by dynamic light scattering (Zetasizer Nano ZS, Malvern Instruments, UK). Detection of the scattered light was carried out at an angle of $173^{\circ}$ at $25^{\circ} \mathrm{C}$. The zeta potential was also measured by laser Doppler microelectrophoresis using a Zetasizer ZS Nano (Zetasizer Nano ZS, Malvern Instruments, UK). All measurements were carried out in triplicate. The results are given as mean \pm standard deviation of the three values obtained.

\section{Nanoparticles morphology}

The morphology of NPs was observed by transmission electron microscopy (Morgagni (TM) FEI 268, Holland). The samples were placed directly on copper grids and allowed to dry at room temperature before observation.

\section{Stability studies at physiological pHs}

Aliquots of all NP formulas whether ionotropic or covalent were studied under two $\mathrm{pH}$ ranges and evaluated by dynamic light scattering (DLS). $\mathrm{pH}$ adjustments were achieved by the dropwise addition of aqueous $\mathrm{NaOH}$ (0.1 M monitored using Jenway pH-meter, UK). Only samples with hazy appearance were analyzed by DLS while samples showing aggregates were not analyzed. All measurements of particle size and Polydispersity Index (PDI) were done in triplicate. 


\section{Drug Loading (DL\%) and Encapsulation Efficiency (EE\%)}

The DL\% and EE\% of the MET-loaded NPs were measured using a modified microfiltration procedure (18). Encapsulated and non-encapsulated portions of MET from the NP dispersion were separated using centrifugal filter devices (Amicon Ultra-0.5, Millipore $\left.{ }^{\circledR}\right)$ with centrifugation at $8000 \mathrm{rpm}$ for $30 \mathrm{~min}$ at $4^{\circ} \mathrm{C}$ using a refrigerated centrifuge (Centurion, UK). The ultrafiltrate resulting from centrifugation was diluted with TRIS base buffer (pH 7.4) and drug content was measured by UV/VIS spectrophotometry (Shimadzu, UV-1800, Japan) at $311 \mathrm{~nm}$. The NPs resulting from centrifugation were freeze-dried (ZirbusVaco-2 Freeze Dryer, Germany). A specific quantity of MET loaded NPs was placed in a mixture of $15 \mathrm{~mL}$ of acetic acid/ absolute ethanol $(10 \%)$ solution and shaken in a water bath at $50^{\circ} \mathrm{C}$ overnight followed by sonication for $30 \mathrm{~min}$ to allow the breakdown of the NPs. Drug content was quantified by UV spectroscopy at the same wavelength described above.

The (EE\%) and (DL\%) were calculated by the following equations:

$$
\begin{gathered}
E E \%=\frac{\text { Total amount of drug in specific volume }- \text { Amount of drug in solution }}{\text { Total amount of drug in specific volume }} \times 100 \\
D L \%=\frac{\text { Total amount of drug in specific weight of NPs }}{\text { Weight of NPS }} \times 100 \%
\end{gathered}
$$

\section{In vitro release studies}

The dialysis membrane method was employed in the MET in vitro release studies. Release tests were carried out in TRIS base buffer ( $\mathrm{pH}$ : 7.4) at $37^{\circ} \mathrm{C}$ for $24 \mathrm{~h}$. A specific volume of the filtered NPs was placed in a dialysis bag (pore size $2.5 \mathrm{~nm}$, molecular weight cut off 12000-14000 Da, Merck) in release media and was shaken using a shaking water bath (Thermo, China) at $100 \mathrm{rpm}$ at $37^{\circ} \mathrm{C}$ for $24 \mathrm{~h}$. At pre-determined time intervals, $2 \mathrm{~mL}$ of sample was withdrawn and replaced with an equal volume of the corresponding fresh media. MET concentration was quantified at $311 \mathrm{~nm}$ using UV/ VIS spectroscopy (Shimadzu UV-1800, Japan) as described above.

\section{Analytical method validation}

Method validation was carried out according to the procedures described by the International Conference on Harmonization (ICH, 2005) (19) using an Ultraviolet-visible spectrophotometer (Shimadzu 1800, Japan). The method involved the measurement of specificity, Limit of Detection (LOD), Limit of Quantitation (LOQ), linearity, range, accuracy, in addition to precision.

\section{Specificity}

Specificity was evaluated by analyzing the UV spectra of MET and the LEC/CHI NPs (without MET) to guarantee that no interference occurred in the absorbance region as a reference for MET quantification. The UV spectra of MET and drug-free LEC/CHI NPs. Absorption spectra did not show any potential interference from the NP constituents at $311 \mathrm{~nm}$.

\section{Limit of Detection (LOD) and the Limit of Quantitation (LOQ)}

LOD and LOQ for MET were estimated by extrapolation of the regression line through Y-axis from a standard curve at low concentration using the following equations:

$$
L O D=\frac{3.3 \times \sigma}{\text { slope }} \quad L O Q=\frac{10 \times \sigma}{\text { slope }}
$$

$\sigma$ : standard deviation of intercept with Y-axis.

The values calculated for LOD and LOQ were $0.01 \mu \mathrm{g} / \mathrm{mL}$ and $0.03 \mu \mathrm{g} / \mathrm{mL}$, respectively.

\section{Linearity and range}

The linearity and range were investigated using MET solutions in TRIS base Buffer $\mathrm{pH} 7.4$ in six different concentration levels ranging from 2-6 $\mu \mathrm{g}$ / $\mathrm{mL}$. A linear relationship was found between the $\mathrm{ab}-$ sorbance and the concentration of MET in the range of 2 to $6 \mu \mathrm{g} / \mathrm{mL}$. The correlation coefficient was 0.9999 indicating excellent linearity $\left(r^{2}>0.999\right)$. The regression parameters for the curve, described by the equation: $\mathrm{y}=\mathrm{ax}+\mathrm{b}$, were $\mathrm{a}=0.1027, \mathrm{~b}=0.0024$ and $r=0.9999(n=6)$ calculated by the least squares method. SD of Intercept was 0.0016 . Therefore, the method is linear for MET within the evaluated ranges.

\section{Accuracy and precision}

The recovery assay was conducted on drugfree NPs dispersions with concentrations of 2, 4, and $6 \mu \mathrm{g} / \mathrm{mL}$, which corresponds to the minimum, medium and maximum values of the previously determined standard calibration curve. Assays were performed in triplicates and the Relative Standard Deviation percentage (RSD\%) values were evaluated. The precision study consisted of the determination of the intermediate precision (assays on different days) and the repeatability (different assays on the same day). The intermediate precision and repeatability were determined with six scans of three MET solutions with known concentrations. Precision levels were calculated by the (RSD\%) from the analytical curves. The intra-day 


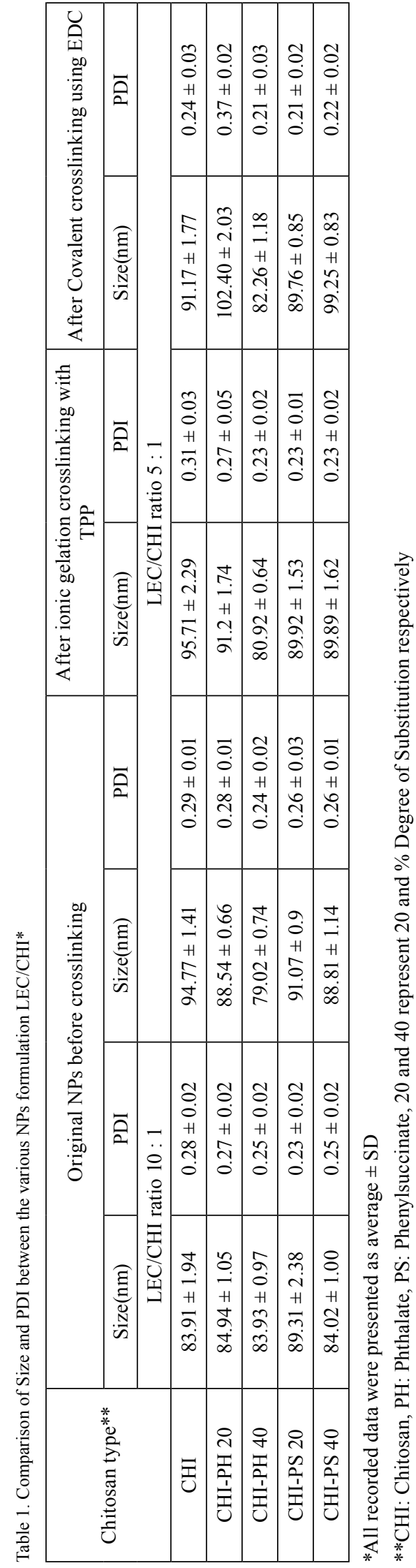

and inter-day relative standard deviation (RSD) values obtained by the proposed method were found to be lower than $2.0 \%$. The accuracy of the method expressed as recovery (\%) was between 99.1 and $100.7 \%$. According to the results obtained, it can be concluded that the UV spectrophotometric method was adequate to quantify MET in the presence of LEC/CHI NPs.

\section{Statistical analysis}

Each experiment was replicated at least 3 times and the values reported are expressed as mean \pm standard deviation (SD). Percent Relative Standard Deviation (RSD\%) was calculated in the analytical method validation. Microsoft Excel Software 2007 (Microsoft Corp., Redmont, WA, USA) was used to create graphs. Statistical comparison was performed using Student's t-test. P-values of less than 0.05 were considered statistically significant.

\section{RESULTS AND DISCUSSION}

During the last two decades, the use of polymeric NPs has been extensively investigated as a drug delivery system. In this article, we introduced chitosan derivatives in the formation of a LEC/CHI assembly and compared it with NPs based on unmodified chitosan. These NPs were generated as a result of the electrostatic interaction between the negatively charged lecithin and the positively charged chitosan or chitosan derivatives resulting in hazy dispersion characteristic of NP dispersions. Following their fabrication, these NPs were further subjected to dual ionic / covalent crosslinking as depicted in Figure 2.

\section{Particle size and zeta potential measurements}

The size and zeta potential of the prepared NPs were measured using Dynamic Light Scattering (DLS) (Table 1). In general, the size was comparable to LEC/CHI previously published data (20). The hydrodynamic diameter in all prepared NPs was less than $100 \mathrm{~nm}$ while the PDI of the measured NPs was below 0.3 in general indicating the uniformity of the size within the prepared dispersions. This small particle size has some advantages including the large surface area and the ability to release the drug easily. After the dual ionic/covalent crosslinking was applied, the size range remained below $100 \mathrm{~nm}$ with PDI less than 0.4. This indicates the formation of condensed NP assemblies following crosslinking independent of the introduction of the larger molecules of chitosan conjugates. The data in Table 1 also indicates that increasing the chitosan ratio from $10: 1$ to $5: 1$ did not affect the NP size. The ratio $5: 1$ was used in the rest of the study to investigate the effect of using different types of conjugated chitosan in addition to chitosan itself.

In all NP formulations, the zeta-potential was positive ranging from 20 to $23 \mathrm{mV}$ before dual crosslinking (Table 2). Comparing chitosan derivative-based NPs to chitosan NPs, 
Table 2. Zeta potential of the various formulations before and after crosslinking*.

\begin{tabular}{|c|c|c|c|c|}
\hline \multirow[t]{2}{*}{ Chitosan type** } & \multicolumn{2}{|c|}{ Zeta potential $(\mathrm{mV})$ before crosslinking } & $\begin{array}{l}\text { Zeta potential }(\mathrm{mV}) \\
\text { after ionic gelation } \\
\text { crosslinking with } \\
\text { TPP }\end{array}$ & $\begin{array}{c}\text { Zeta potential (mV) } \\
\text { after Covalent } \\
\text { crosslinking using } \\
\text { EDC }\end{array}$ \\
\hline & $10: 1$ & $5: 1$ & \multicolumn{2}{|c|}{$5: 1$} \\
\hline $\mathrm{CHI}$ & $23.4 \pm 0.5$ & $23.1 \pm 0.7$ & $20.6 \pm 1.6$ & $14.4 \pm 0.2$ \\
\hline CHI-PH 20 & $24.7 \pm 1.4$ & $22.0 \pm 0.5$ & $16.9 \pm 1.1$ & $14.0 \pm 1.0$ \\
\hline CHI-PH 40 & $22.1 \pm 1.8$ & $20.1 \pm 0.9$ & $16.8 \pm 1.5$ & $13.2 \pm 0.9$ \\
\hline CHI-PS 20 & $23.7 \pm 1.7$ & $20.8 \pm 0.9$ & $17.4 \pm 1.4$ & $17.3 \pm 1.0$ \\
\hline CHI-PS 40 & $25.1 \pm 1.8$ & $21.0 \pm 0.6$ & $18.5 \pm 2.0$ & $16.2 \pm 1.7$ \\
\hline
\end{tabular}

*All recorded data were presented as average $\pm \mathrm{SD}$

**CHI: Chitosan, PH: Phthalate, PS: Phenylsuccinate, 20 and 40 represent 20 and \% Degree of Substitution respectively.

it can be noticed that the degree of substitution decreased the zeta potential when phthalate or phenylsuccinate conjugates were introduced to the NP assembly, due to the decrease in chitosan amino groups that were replaced with carboxyl groups upon conjugation. In addition, crosslinking with TPP followed by EDC decreased the NP surface positive charge to reach $13 \mathrm{mV}$ in Chitosan phthalate and to a lesser extent in the phenylsuccinate. This can be explained by the involvement of the amine of the chitosan and carboxyl groups in the chitosan derivative in the crosslinking step, whether it was electrostatic by TPP or covalent crosslinking by EDC.

It has been previously reported that the incorporation of high phospholipid content will emphasize the amount of chitosan associated with the surface of the NP (21). However, the change of zeta potential observed was limited when the ratio of lecithin to chitosan was increased from $10: 1$ to $5: 1$.

\section{Stability study at physiological pHs}

The stability of the fabricated NPs was studied at $\mathrm{pH} 6.8$ and 7.4. As can be seen in Table 3, the NPs preparation pHs ranged between 2.47 and 2.64. The use of unmodified chitosan in the NP assembly showed aggregation at $\mathrm{pH} 7.4$ before and after crosslinking. At $\mathrm{pH}$ above 6.5 (pKa of chitosan), aggregation can be observed due to the deprotonation of chitosan amine groups $(11,22)$. The use of covalent crosslinking of chitosan derivative provided stable NPs when exposed either to $\mathrm{pH} 6.8$ or 7.4. Although we mentioned above that some interactions between chitosan and crosslinkers (evidenced by a decrease in zeta potential) were observed (Table 2), it seems that the configuration of crosslinking in unmodified chitosan was insufficient to provide a stable NP which resulted in aggregation. While in the case of chitosan derivatives, the stability could be attributed to the effective interaction between the

Table 3. Stability assessments of LEC/CHI NPs physiological pHs*

\begin{tabular}{|c|c|c|c|c|c|c|c|}
\hline \multirow{3}{*}{$\begin{array}{l}\text { Chitosan } \\
\text { type** }\end{array}$} & \multirow{3}{*}{$\begin{array}{c}\text { Preparation } \\
\mathrm{pH}\end{array}$} & \multirow{2}{*}{\multicolumn{2}{|c|}{$\begin{array}{l}\text { Original NPs before } \\
\text { crosslinking } \\
\text { Size }(\mathrm{nm}) \\
(\mathrm{PDI})\end{array}$}} & \multirow{2}{*}{\multicolumn{2}{|c|}{$\begin{array}{c}\text { After ionic gelation } \\
\text { crosslinking with TPP } \\
\text { Size }(\mathrm{nm}) \\
(\mathrm{PDI})\end{array}$}} & \multirow{2}{*}{\multicolumn{2}{|c|}{$\begin{array}{c}\text { After Covalent crosslinking } \\
\text { using EDC } \\
\text { Size }(\mathrm{nm}) \\
(\mathrm{PDI})\end{array}$}} \\
\hline & & & & & & & \\
\hline & & pH 6.8 & $\mathrm{pH} 7.4$ & pH 6.8 & $\mathrm{pH} 7.4$ & pH 6.8 & $\mathrm{pH} 7.4$ \\
\hline $\mathrm{CHI}$ & 2.64 & $\begin{array}{c}102.8 \pm 3.55 \\
(0.28 \pm 3.56)\end{array}$ & Aggregation & $\begin{array}{l}94.68 \pm 2.84 \\
(0.33 \pm 0.06)\end{array}$ & Aggregation & $\begin{array}{c}99.56 \pm 8.12 \\
(0.36 \pm 0.07)\end{array}$ & Aggregation \\
\hline CHI-PH 20 & 2.47 & $\begin{array}{l}151.9 \pm 13.77 \\
(0.64 \pm 0.06)\end{array}$ & $\begin{array}{c}91.37 \pm 2.02 \\
(0.29 \pm 0.05)\end{array}$ & $\begin{array}{c}142.0 \pm 4.8 \\
(0.58 \pm 0.11)\end{array}$ & $\begin{array}{c}127.7 \pm 6.05 \\
(0.36 \pm 0.05)\end{array}$ & $\begin{array}{l}119.0 \pm 2.25 \\
(0.27 \pm 0.01)\end{array}$ & $\begin{array}{l}130.1 \pm 4.89 \\
(0.42 \pm 0.06)\end{array}$ \\
\hline CHI-PH 40 & 2.62 & $\begin{array}{l}89.56 \pm 2.97 \\
(0.27 \pm 0.03)\end{array}$ & $\begin{array}{l}97.42 \pm 2.39 \\
(0.29 \pm 2.39)\end{array}$ & Aggregation & Aggregation & $\begin{array}{c}116.2 \pm 7.8 \\
(0.50 \pm 0.09)\end{array}$ & $\begin{array}{r}105.6 \pm 3.40 \\
(0.36 \pm 0.00)\end{array}$ \\
\hline CHI-PS 20 & 2.49 & $\begin{array}{c}105.8 \pm 7.8 \\
(0.42 \pm 0.13)\end{array}$ & $\begin{array}{r}92.71 \pm 2.06 \\
(0.38 \pm 0.01)\end{array}$ & Aggregation & $\begin{array}{l}142.0 \pm 4.87 \\
(0.58 \pm 0.11)\end{array}$ & $\begin{array}{c}99.86 \pm 2.78 \\
(0.35 \pm 0.06)\end{array}$ & $\begin{array}{c}101.6 \pm 6.82 \\
(0.39 \pm 0.06)\end{array}$ \\
\hline CHI-PS 40 & 2.57 & $\begin{array}{c}148.7 \pm 9.45 \\
(0.53 \pm 0.07)\end{array}$ & $\begin{array}{c}104.8 \pm 5.5 \\
(0.36 \pm 0.05)\end{array}$ & $\begin{array}{c}151.05 \pm 9.86 \\
(0.52 \pm 0.16)\end{array}$ & Aggregation & $\begin{array}{l}115.6 \pm 13.46 \\
(0.34 \pm 0.05)\end{array}$ & $\begin{array}{c}123.1 \pm 3.95 \\
(0.34 \pm 0.03)\end{array}$ \\
\hline
\end{tabular}

*All recorded data were presented as average $\pm \mathrm{SD}$

**CHI: Chitosan, PH: Phthalate, PS: Phenylsuccinate, 20 and 40 represent 20 and \% Degree of Substitution respectively 
Table 4. Characterization of MET loaded NPs*.

\begin{tabular}{|c|c|c|c|c|}
\hline \multirow{2}{*}{ Chitosan type** } & \multicolumn{2}{|c|}{ Original NPs before crosslinking } & \multicolumn{2}{c|}{ After covalent crosslinking using EDC } \\
\cline { 2 - 5 } & Size $(\mathrm{nm})$ & $(\mathrm{PDI})$ & Size $(\mathrm{nm})$ & $(\mathrm{PDI})$ \\
\hline CHI & $112.9 \pm 0.83$ & $0.27 \pm 0.02$ & $119.6 \pm 3.63$ & $0.34 \pm 0.05$ \\
\hline CHI - PH 20 & $99.97 \pm 1.41$ & $0.30 \pm 0.05$ & $111.4 \pm 2.54$ & $0.37 \pm 0.02$ \\
\hline CHI - PH 40 & $101.4 \pm 3.66$ & $0.30 \pm 0.04$ & $97.98 \pm 1.65$ & $0.24 \pm 0.02$ \\
\hline CHI - PS 20 & $106.4 \pm 1.51$ & $0.33 \pm 0.03$ & $439.9 \pm 37.44$ & $0.63 \pm 0.17$ \\
\hline CHI - PS 40 & $104.9 \pm 1.76$ & $0.28 \pm 0.02$ & $109.2 \pm 1.65$ & $0.24 \pm 0.03$ \\
\hline
\end{tabular}

*All recorded data were presented as average $\pm \mathrm{SD}$

**CHI: Chitosan, PH: Phthalate, PS: Phenylsuccinate, 20 and 40 represent 20 and \% Degree of Substitution respectively

carboxyl group present in the phthalate and phenylsuccinate derivative with the two crosslinkers TPP and EDC (11). It has been previously reported that the introduction of a carboxylic group to chitosan backbone and in presence of TPP and EDC in the preparation media, resulted in NP stabilization due to phosphoramide group formation via a mixed anhydride intermediate formation. The formation of a phosphoramide group facilitated the maintenance of repulsion between NPs and prevented the aggregation upon exposure to $\mathrm{pHs}$ higher than $6.5(\mathrm{pKa}$ of chitosan) (11). It should be pointed out that the involvement of several components within the crosslinked NP assembly did not allow the elucidation and confirmation of the type of interaction between the various components by infrared spectroscopy for example.

\section{Characterization of drug-loaded NPs Particle size and PDI determination of drug-loaded NPs}

As can be seen in Table 4, there was a slight increase in the size of the loaded NPs of the various formulations (size less than $120 \mathrm{~nm}$ ) and PDI less than 0.5 before and after crosslinking. This can be attributed to the high drug: polymer ratio $(1: 1)$ used in drug loading and to the potential interaction between the drug and the other components of
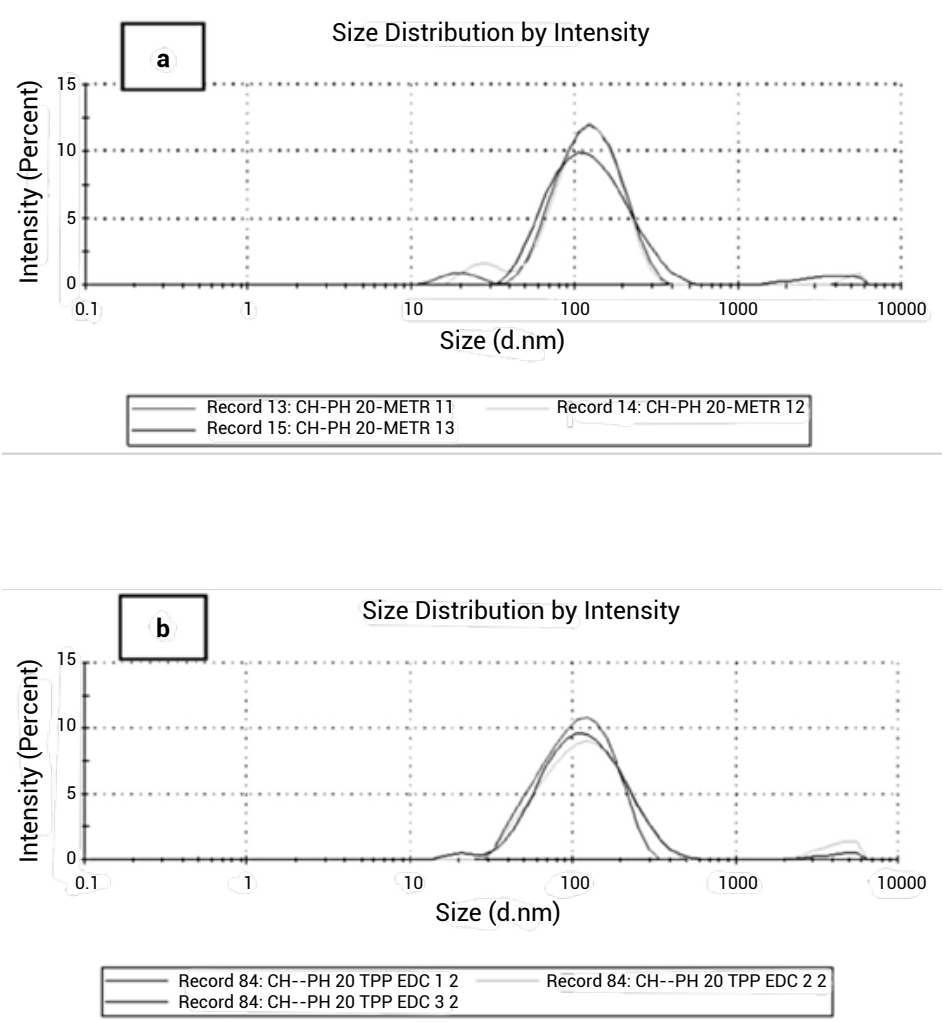

Figure 3. Particle size distribution obtained by DLS of MET loaded Lecithin-chitosan phthalate NPs with DS $20 \%$ (a) before dual crosslinking (b) after dual crosslinking. 


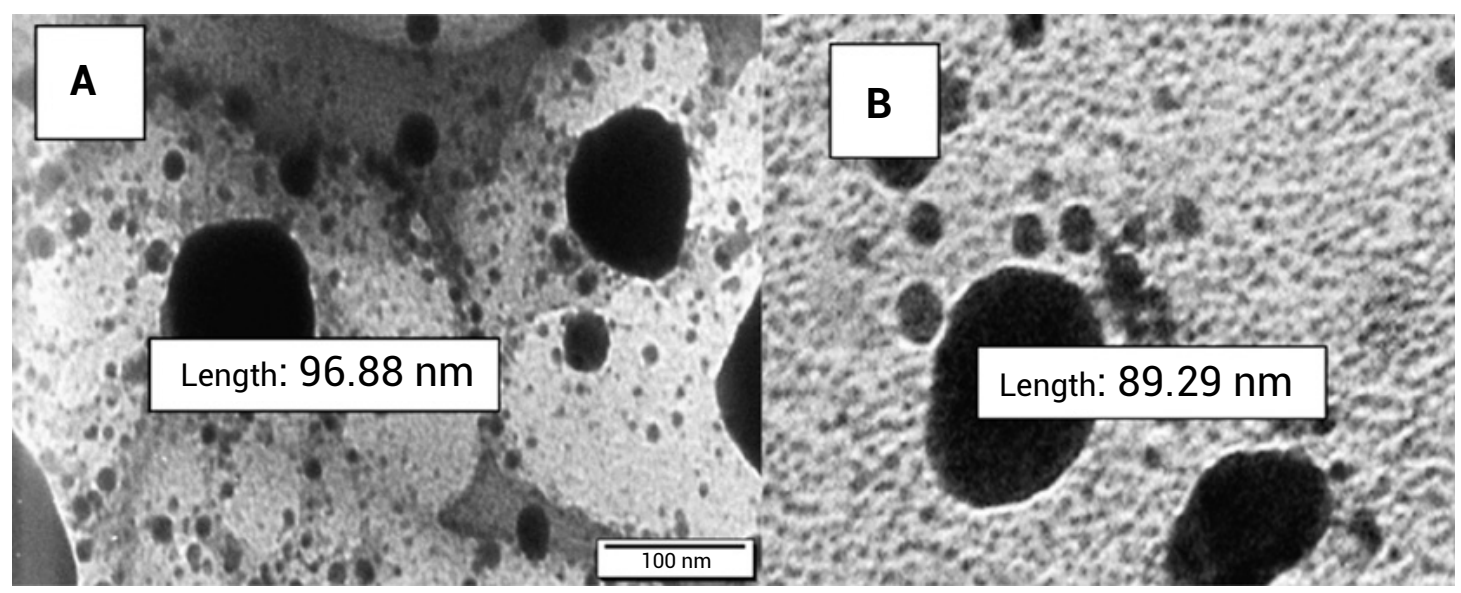

Figure 4. TEM of Lecithin-chitosan phthalate NPs with DS $40 \%$ (a) before dual crosslinking (b) after dual crosslinking.

the NPs like lecithin or chitosan polymeric chains. Table 4 shows also the large increase in the size of chitosan Phenylsuccinate (20\% DS). The low degree of substitution did not allow efficient crosslinking since phenylsuccinate as a substituent has been previously postulated to result in one-sided rather than two-sided crosslinking (11). This type of interaction may result in non-efficient packing of the polymeric parts within the NP assembly following MET loading. Figure 3 also illustrates the NP size distribution for $\mathrm{LEC} / \mathrm{CHI}$-phthalate with $20 \% \mathrm{DS}$ as generated by DLS with minimum changes in the hydrodynamic diameter after dual crosslinking.

\section{Morphological analysis by TEM}

TEM was used to depict the morphology of the prepared NPs. TEM images were captured before and after covalent crosslinking for LecithinChitosan Phthalate with DS 40\% NPs before and after dual crosslinking (Figure 4). The TEM photographs showed that the synthesized NPs were almost spherical. There was no significant change in the size of the NPs before and after crosslinking. The hydrodynamic diameter is in correlation with the reported average size measured by DLS.

\section{Determination of Drug Loading (DL\%) and Encapsulation Efficiency (EE\%)}

The DL\% and EE\% were determined for MET loaded NPs using a ratio of $1: 1$ (LEC: MET) as seen in Table 5. This ratio was selected during the optimization studies (data is not shown). It is clear from Table 5 that drug DL\% increased significantly after the covalent crosslinking was performed in all formulations $(\mathrm{p}<0.05)$. The highest DL\% was 26.59 for CHI-PH 40 and the lowest was 9.18 for unsubstituted chitosan. It can be noticed that increasing the DS increased the DL\%. This can be attributed to the increased chance of crosslinking with increasing DS which allowed more drugs to be entrapped during the loading process. It has been reported that LEC/CHI are best used to encapsulate lipophilic drugs rather than hydrophilic drugs due to the lipophilic nature of NPs (2). In this study, we were able to achieve high DL\% with a slightly water-soluble drug-like MET which can be attributed to the application of the dual crosslinking procedure involved.

Table 5. Drug Loading (DL\%) and Encapsulation Efficiency (EE\%) for MET loaded NPs before and after covalent crosslinking*.

\begin{tabular}{|c|c|c|c|c|c|c|}
\hline $\begin{array}{c}\text { Chitosan } \\
\text { type** }\end{array}$ & $\begin{array}{c}\text { DL\% } \\
\text { before dual } \\
\text { crosslinking }\end{array}$ & $\begin{array}{c}\text { DL\% after dual } \\
\text { crosslinking }\end{array}$ & p-value & $\begin{array}{c}\text { EE\% } \\
\text { before dual } \\
\text { crosslinking }\end{array}$ & $\begin{array}{c}\text { EE\% after dual } \\
\text { crosslinking }\end{array}$ & p-value \\
\hline CHI & $4.23 \pm 0.38$ & $9.18 \pm 0.17$ & 0.01 & $56.4 \pm 1.85$ & $65.49 \pm 1.43$ & 0.09 \\
\hline CHI-PH 20 & $5.07 \pm 0.23$ & $11.72 \pm 0.37$ & 0.01 & $78.45 \pm 1.04$ & $66.65 \pm 1.22$ & 0.02 \\
\hline CHI-PH 40 & $5.12 \pm 0.01$ & $26.59 \pm 0.36$ & 0.00 & $79.39 \pm 1.83$ & $71.46 \pm 0.46$ & 0.08 \\
\hline CHI-PS 20 & $7.10 \pm 0.52$ & $14.22 \pm 0.80$ & 0.04 & $56.03 \pm 1.79$ & $56.74 \pm 0.83$ & 0.42 \\
\hline CHI-PS 40 & $5.34 \pm 0.41$ & $16.9 \pm 0.62$ & 0.01 & $60.66 \pm 1.66$ & $73.82 \pm 1.72$ & 0.04 \\
\hline
\end{tabular}

*All recorded data were presented as average $\pm \mathrm{SD}$

**CHI: Chitosan, PH: Phthalate, PS: Phenylsuccinate, 20 and 40 represent 20 and \% Degree of Substitution respectively 

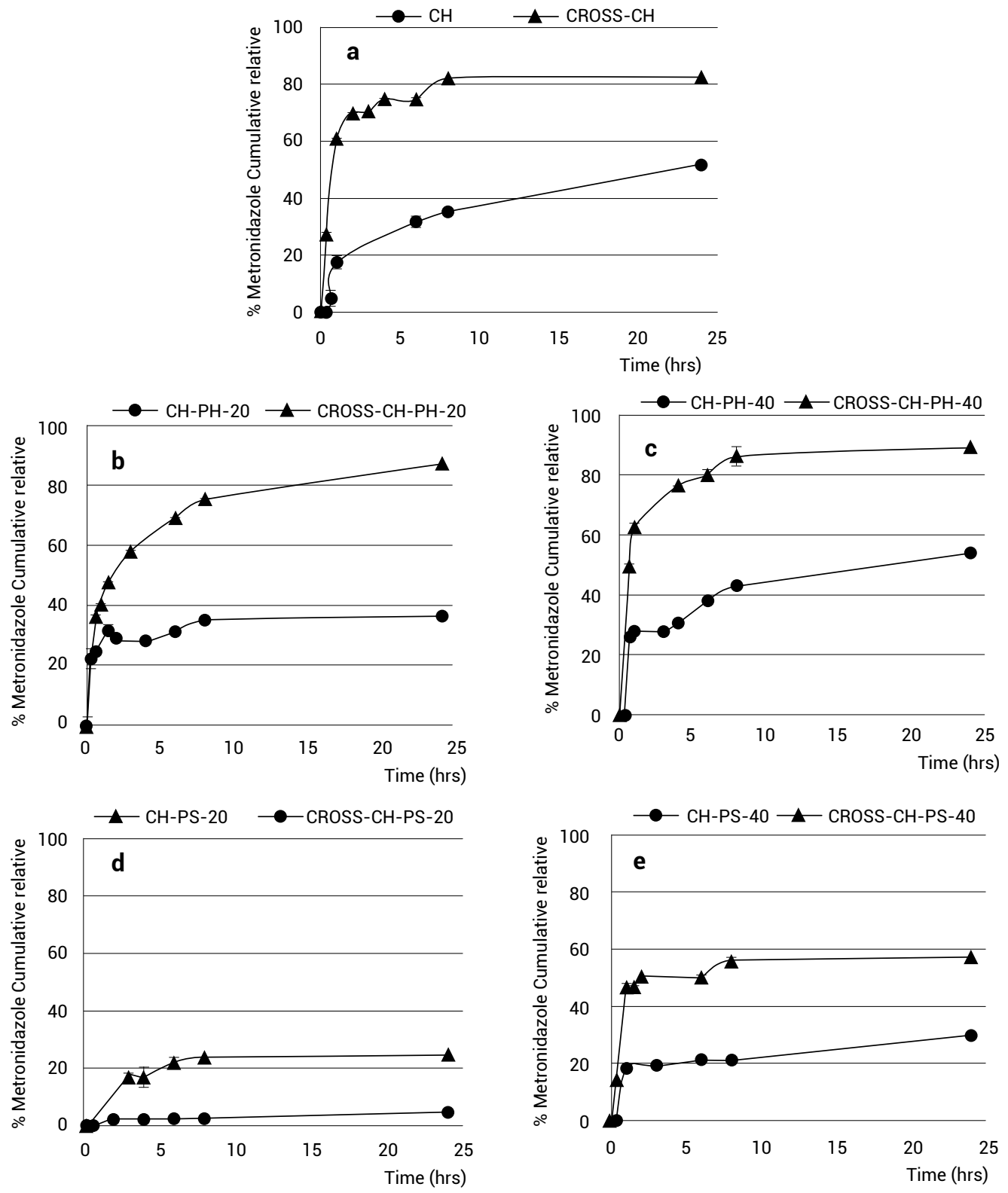

Figure 5. Release profiles of LEC/CHI NPs using Chitosan (a), Chitosan Phthalate 20\% (b), chitosan phthalate 40\% (c), Chitosan phenylsuccinate $20 \%$ (d), chitosan phenylsuccinate $40 \%$ (e) before crosslinking $(\bullet)$ compared to their respective dual crosslinked $\operatorname{NPs}(\boldsymbol{\Delta})$.

The high EE\% and DL\% obtained with lecithin/chitosan NPs may be related to some affinity between lecithin and MET.

According to published studies, drug loading reflects the mass of the drug in NPs and its content depends mainly on the physical and chemical properties of the polymeric material and other processing factors (23). It should be emphasized that physical and electrostatic interaction will usually result in low drug-loading efficiency, as seen in the case of unmodified chitosan and TPP in the fabricated NPs, while covalent and coordinate bonds will usually give high drug-loading values. The high DL\% can be attributed to the increase in the space capacity of the NP assembly resulting from covalent crosslinking between the chitosan derivative and lecithin molecules.

Among the important factors to be considered in drug encapsulation is the solubility of the drug within the solvent and its ionicity and drug 
Table 6. Intra-day and inter-day precision data of UV spectrophotometric method for MET in LEC/CHI NPs.

\begin{tabular}{|c|c|c|c|}
\hline \multicolumn{4}{|c|}{ Accuracy } \\
\hline Nominal concentration $(\mu \mathrm{g} / \mathrm{mL})$ & Mean concentration found* $(\mu \mathrm{g} / \mathrm{mL})$ & $\operatorname{RSD}(\%)^{* *}$ & Accuracy $(\%)$ \\
\hline 2 & 2.09 & 0.6 & 99.8 \\
\hline 4 & 4.06 & 0.7 & 100.7 \\
\hline 6 & 6.1 & 0.7 & 99.1 \\
\hline Mean recovery $\%$ & & & 99.7 \\
\hline \multicolumn{4}{|c|}{$\begin{array}{l}\text { Precision } \\
\end{array}$} \\
\hline Precision & Experimental concentration $(\mu \mathrm{g} / \mathrm{mL})$ & Recovery (\%) & $\operatorname{RSD}(\%) * *$ \\
\hline $\operatorname{Intra-day}(\mathrm{n}=6)$ & 5.25 & 99.4 & 0.6 \\
\hline \multicolumn{4}{|l|}{ Inter day } \\
\hline Day $1(\mathrm{n}=3)$ & 5.09 & 100.7 & 0.9 \\
\hline Day $2(n=3)$ & 5.02 & 99.8 & 1.0 \\
\hline Day $3(\mathrm{n}=3)$ & 5.16 & 101.1 & 1.2 \\
\hline Mean $(n=9)$ & 5.09 & 100.5 & 1.1 \\
\hline
\end{tabular}

*Mean of three replicates

$* * \mathrm{RSD}=$ Relative standard deviation

lipophilicity (24). It has been reported previously that LEC/CHI NPs are best used for lipophilic drugs like progesterone and clobetasol due to increased favorable interactions between these drugs and the lipid carrier $(2,9)$. Despite the fact that MET contains both lipophilic and hydrophilic functional groups within its small molecular structure it showed increased $\mathrm{DL} \%$ and $\mathrm{EE} \%$ following covalent crosslinking. This can be attributed to the introduction of the hydrophobic moieties of phthalate and phenylsuccinate which limit the chance of drug diffusion following loading. In addition, covalent crosslinking will decrease the chance of water penetration to the interior of the NP, thus decreasing drug permeation (25).

\section{In vitro release studies}

Figure 5 shows the release profiles of all NP formulae before and after covalent crosslinking. It can be observed that all formulae showed rapid release in the first 2 hours due to the release of the adsorbed drug on the NP surface or due to weak binding within the NP. Following that, a plateau can be seen in all the release profiles. Also, the crosslinked formula showed a higher $\%$ release compared to the uncrosslinked formula. This can be attributed to the higher drug loading as mentioned in the previous section and to the interaction between the slightly water-soluble MET and the hydrophobic moieties introduced by chitosan grafting. In addition, the covalent crosslinking decreased the chance for drug leaching outside the NP compared to uncrosslinked NPs (11-12). As seen in Figure 5d, the phenylsuccinate based formula with a $20 \%$ degree of substitution showed a decrease in $\%$ cumulative release for the crosslinked formula compared to the uncrosslinked formula. This can be attributed to the low DS and weak capability of the phenylsuccinate substituent to form two-sided crosslinking, which correlates well with a similar finding that has been previously reported (11). The highest $\%$ cumulative release reached only $80 \%$, this can be explained by the absence of lipid enzymes within the release media which leads to the degradation of the lecithin layer thus allowing drug permeation (13).

Table 6 shows summary results of the accuracy and precision of MET using UV / VIS spectroscopy. In addition, the linear regression analysis of the calibration curve of MET and the UV/VIS spectra for pure MET and LEC/CHI nanoparticles (without MET) are depicted in Figure 6. These results are in compliance with the ICH guideline (19).

\section{CONCLUSION}

Our results highlighted novel lecithin/chitosanbased NPs using chitosan conjugates which can be fabricated to be used for the encapsulation of MET. This type of conjugation allows the manipulation of the lipophilic/hydrophilic interaction within the NPs. Additionally, the degree of substitution of each conjugate has a tremendous effect on the MET loading and encapsulation efficiency. Most probably these changes are related to a change in the lipophilicity of the chitosan when conjugated. Results of the present study indicate that dual crosslinking allows the preparation of stable NPs at physiological pHs. 
(a)

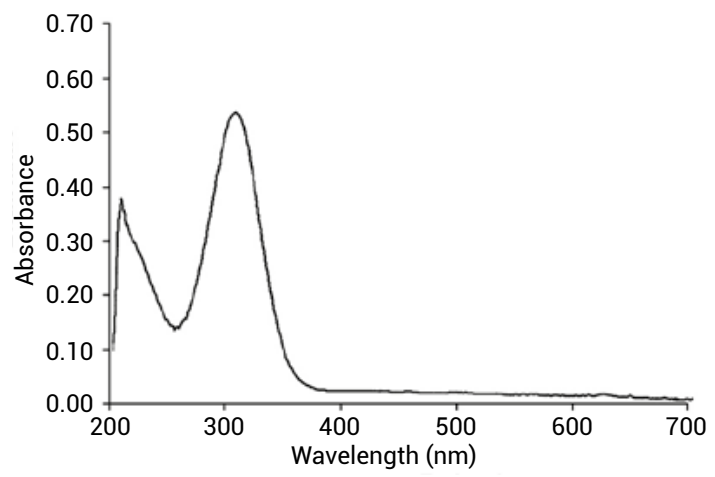

(b)

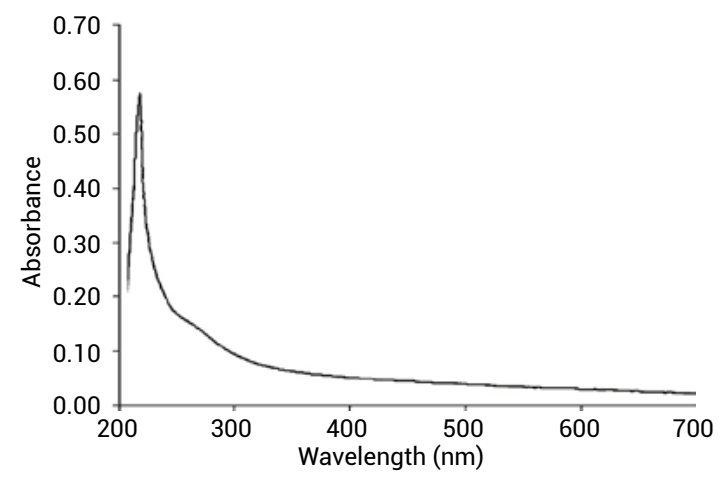

(c)

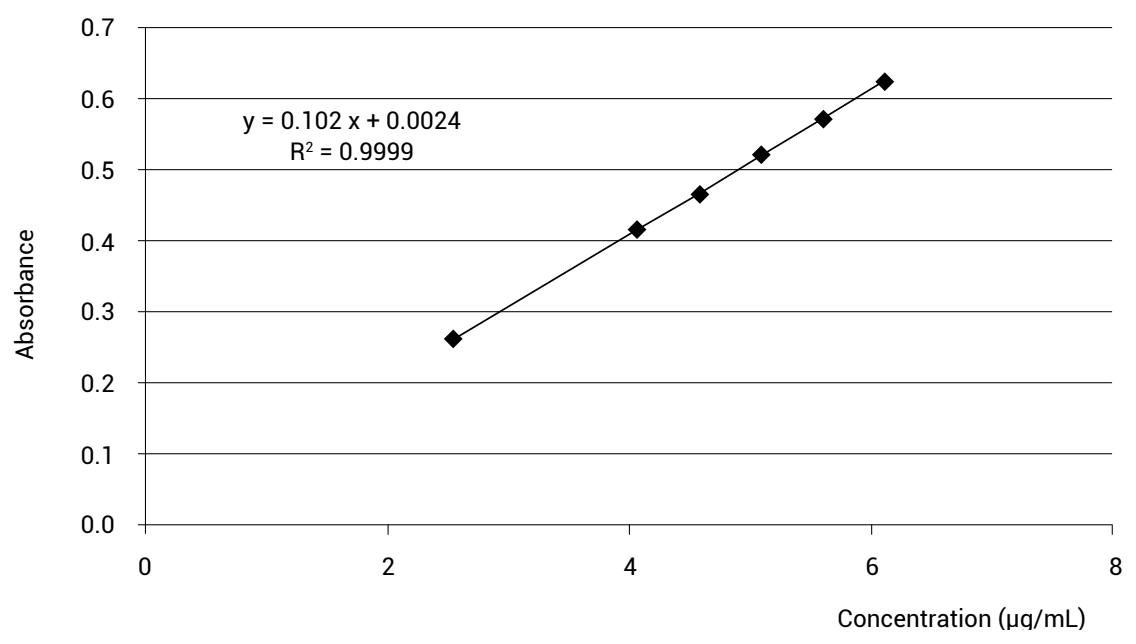

Figure 6. UV/VIS spectra for: (a) pure metronidazole and (b) LEC/CHI nanoparticles (without MET). (c) Linear regression analysis of the calibration curve of metronidazole UV/VIS spectrophotometer.

Applying TPP/EDC dual crosslinking to LEC/CHI NPs is found to improve drug loading properties. These findings also illustrate the possibility of enhanced release properties when dual crosslinking is applied to the NPs.

\section{Acknowledgments}

The authors would like to acknowledge Prof. Mutasem Taha (the University of Jordan, Faculty of Pharmacy) for useful discussions of the results reported in this work. We are very thankful to Dr. Iman Khalil for proofreading this manuscript. The authors would like to thank Ms. Rahma Khirfan (Ahliyya Amman University, Faculty of Pharmacy) for her technical assistance.

\section{Funding information}

The authors gratefully acknowledge the financial support from Ahliyya Amman University
(Deanship of Scientific Research), Scientific Research Project grant no. (43/2017-2018).

\section{Conflict of interest}

The authors report no conflicts of interest. The authors alone are responsible for the content and writing of this article.

\section{REFERENCES}

1. Parveen S., Misra R., Sahoo S.K.: Nanomedicine 8, 147 (2012).

2. Sonvico F., Cagnani A., Rossi A., Motta S., Di Bari M.T., et al.: Int. J. Pharm. 324, 67 (2006).

3. Dmour I. Taha M.O.: Natural and semisynthetic polymers in pharmaceutical nanotechnology. In Organic Materials as Smart Nanocarriers for Drug Delivery; pp. 35-100, William Andrew Publishing, Oxford 2018. 
4. Garg U., Chauhan S., Nagaich U., Jain N.: Adv. Pharm. Bull. 9, 195 (2019).

5. Naskar S., Koutsu K., Sharma S.J.: J. Drug Target. 27, 379 (2019).

6. Barbieri S., Buttini F., Rossi A., Bettini R., Colombo P., et al.: Int. J. Pharm. 491, 99 (2015).

7. Chadha R., Gupta S., Pathak N.: Drug Dev. Ind. Pharm. 38, 1538 (2012).

8. Liu L., Zhou C., Xia X., Liu Y.: Int. J. Nanomedicine 11, 761 (2016).

9. Šenyiğit T., Sonvico F., Barbieri S., Özer Ö., Santi P., Colombo P.: J. Control. Rel. 142, 368 (2010).

10. Le N., Cao V.D., Nguyen T., Le T., Tran T.T., Hoang Thi T.T.: Int. J. Mol. Sci. 20, 4706 (2019).

11. Dmour I., Taha M.O.: Int. J. Pharm. 529, 15 (2017).

12. Saeed R.M., Dmour I., Taha M.O.: Front. Bioeng. Biotechnol. 8, 21 pages (2020).

13. Barthelmes J., Dünnhaupt S., Hombach J., Bernkop-Schnürch A.: Drug Deliv. 18, 613 (2011).

14. Soliman G.M., Zhang Y.L., Merle G, Cerruti M., Barralet J.: Eur. J. Pharm. Biopharm. 88, 1026 (2014).

15. Vila-Sanjurjo C., Hembach L., Netzer J., Remuñán-López C., Vila-Sanjurjo A., Goycoolea F.M.: J. Colloid Interface Sci. 578, 171 (2020).
16. Chien W.Y.: J. Pharm. Sci. Technol. 38, 32 (1984).

17. Aiedeh K., Taha M.: Arch. Pharm. (Weinheim) 332, 103 (1999).

18. Li H., Zhao X., Ma Y., Zhai G, Li L., Lou H.: J. Control. Release 133, 238 (2009).

19. International Conference on Harmonization (ICH) Validation of analytical procedures: Text and methodology (Q2R1), Geneva (2005).

20. Tan Q., Liu W., Guo C., Zhai G.: Int. J. Nanomedicine 6, 1621 (2011).

21. Prego C., Fabre M., Torres D., Alonso M.J.: Pharm. Res. 23, 549 (2006).

22. Shen S., Wu Y., Liu Y., Wu D.: Int. J. Nanomedicine 12, 4085 (2017).

23. Souza M.P., Vaz A.F.M., Correia M.T.S, Cerqueira M.A., Vicente A.A.: Food Bioproc. Tech. 7, 1149 (2014).

24. Cheow W.S., Hadinoto K.: Colloids Surf. B Biointerfaces 85, 214 (2011).

25. Barbieri S., Sonvico F., Bouchemal K., Ponchel G., Colombo P.: Tamoxifen loaded autoassembled nanoparticles for oral delivery: cytotoxicity and permeability studies. In Nanoformulations. Gordon J.T., Reginald B.H. (Eds.), pp. 70-71 (2011). 